less than 32 weeks' gestation (with an out-ofphase response) were all receiving morphine, this was indeed the case: nine of the 10 infants described were receiving morphine by intravenous infusion $(10 \mu \mathrm{g} / \mathrm{kg} /$ hour $)$ as per unit policy. However, only one of the infants with an in-phase relation were receiving inotropic support at the start of their wave episodes. As we suggested, it would appear strange that electromechanical causes could be stopped by merely gently touching the infant.

Infants with overt seizures have been captured many times on our continuous computerised physiological monitoring system, though none have displayed a regular cyclical pattern in heart rate and blood pressure during a convulsion. Seizures were noted in four of the infants described, though only one demonstrated overt seizures during a wave episode. Since the paper was submitted, a further infant has been investigated by 24 hour EEG during a prolonged period of blood pressure waves: no evidence of seizure activity was recorded.

\section{Adverse experiences in an Exosurf treated group}

EDITOR,- The results of recent trials using artificial surfactant for premature neonates with hyaline membrane disease have yielded convincing evidence of efficacy, and are likely to lead to widespread use. ${ }^{1-4}$ We have used Exosurf (Wellcome) in 54 babies as part of the OSIRIS multicentre trial ${ }^{5}$ and were concerned to witness some adverse experiences in a proportion of these babies. We noted an increase in the incidence of lobar collapse and consolidation and blocked endotracheal tubes, resulting in clinical and radiological deterioration. While we would emphasise that it would not be proper to draw any conclusions from our observations, we suggest that they do give rise to some cause for further investigation.

We have seen this problem in babies of varying weights and gestation, not just very low birthweight babies. Decreasing the rate of administration of Exosurf did not seem to improve tolerance and the large volume administered, $5 \mathrm{ml} / \mathrm{kg}$, seemed to be a factor. Because of our concern over the acute deteriorations coinciding with the administration of Exosurf we have looked more closely at the outcome in our Exosurf treated group and compared various outcome measures with a historical group, treated before we entered the OSIRIS trial, of babies matched for birth weight, gestation, and $\mathrm{A} / \mathrm{a}$ ratio.

We found that the babies treated with Exosurf were ventilated for a significantly longer period of time (7 days $v 4.5$ days for the control group) and spent longer in oxygen (12 days $v 8$ days). There was a significantly greater incidence of intraventricular haemorrhage (IVH) (grade III or worse) in the treated group. There was also an increased incidence, albeit not statistically significant at the $5 \%$ level, in pneumothorax, patent ductus arteriosus (PDA), upper lobe consolidation, and pulmonary haemorrhage in the Exosur treated group compared with the control population (table).

Our first impressions concerning numerous adverse short term experiences have been supplemented by the conduct of a casecontrol comparison which also showed that the Exosurf treated babies had a greater duration of ventilator treatment and a longer period in oxygen. The incidence of grade III IVH was also significantly greater in the Exosurf treated group.
Some outcome measures for the infants studied; data are medians or incidences (\%)

$\begin{array}{lll} & \text { Control } & \text { Exosurf } \\ \text { Outcome measures } & \text { group } \\ (n=50) & (n=50)\end{array}$

\begin{tabular}{llc}
\hline Days in IPPV & & \\
Days in oxygen & $8 \cdot 5$ & $7^{\star \star}$ \\
PDA & $1(2)$ & $12^{\star}$ \\
IVH & $3(4)$ & $9(18)$ \\
Pneumothorax & $5(10)$ & $9(18)$ \\
Consolidation & $3(6)$ & $7(14)$ \\
Pulmonary haemorrhage & $0(0)$ & $3(6)$
\end{tabular}

IPPV $=$ intermittent positive pressure ventilation ${ }^{\star \star} \mathrm{p}<0.01 ;{ }^{\star} \mathrm{p}<0.05$.

It is important to bear in mind that our observations take the form of a relatively small case-control study, as compared with the finding of the large multicentre randomised trials that have reported favourably upon the properties of Exosurf. It is possible that our observations are biased because of the lack of randomisation or of exhibiting a type I statistical error on account of chance. However, it may be that the overall beneficial effect discovered in the large multicentre trials hides a group of babies susceptible to an adverse experience with this particular surfactant, although it is possible that some aspect of our management regimen rendered our patients susceptible to an adverse response to Exosurf. It is, for instance, our practice to use muscle relaxants almost routinely in ventilated preterm babies and it is possible that the absence of spontaneous respiratory efforts had an adverse effect on the distribution of surfactant within the lung. If there is a subgroup of babies, distinguished perhaps by the management protocol to which they are subjected, who actually do worse with Exosurf treatment then it is obviously very important for them to be distinguished from the majority who, according to published trials, will benefit.

$$
\begin{array}{r}
\text { S L CHATFIELD } \\
\text { E J KELLY } \\
\text { P R F DEAR } \\
\text { Academic Unit of Paediatrics and Child Health, }
\end{array}
$$
St Fames's University Hospital,
Leeds LS9 $7 T F$

1 Phibbs RH, Balland RA, Clements JA, et al. Initial clinical trial of Exosurf, a protein-free synthetic surfactant, for the prophylaxis and early treatment of hyaline membrane disease. Pediatrics 1991; 88: 1-9.

2 Long WA, Thompson $T$, Swindell $H$, Long WA, Thompson T, Swindell H, two rescue doses of a synthetic surfactant on two rescue doses of a synthetic surfactant on
mortality in 700-1300 $\mathrm{g}$ infants with RDS. mortality in 700-1300 $\mathrm{g}$ infa

3 Geides J, Cook L, Beaumont E, Corbett A, Long W, and the US Exosurf Neonatal Study Group $I$ and II. Effects of these versus one prophylactic doses of Exosurf Neonatal in 700-1100 g infants. Pediatr Res 1991; 29: 214A

4 Long W, Corbett A, Cotton R et al, the American Exosurf Neonatal Study Group I, and the Canadian Exosurf Neonatal Study Group. A controlled trial of synthetic surfactant in infants weighing $1250 \mathrm{~g}$ or more with respiratory
distress syndrome. $N$ Engl $\mathcal{f}$ Med 1991; 325: distress syn

5 The OSIRIS collaborative group. Early versus delayed neonatal administration of a synthetic surfactant - the judgement of OSIRIS. Lancet 1992; 340: 1363-9.

\section{Enterococcus faecium meningitis}

EDIToR,-Neonatal meningitis is a medical emergency in which prompt diagnosis and treatment are of paramount importance. Although $\beta$ haemolytic streptococci of Lancefield group B and Escherichia coli are most commonly responsible, infection with other agents is occasionally encountered. Enterococci are reported as causing neonatal sepsis with increasing frequency, ${ }^{1}$ and their antibiotic sensitivities differ significantly from more common neonatal pathogens. We wish to report two cases of neonatal meningitis in preterm infants caused by Enterococcus faecium.

\section{Case reports}

CASE 1

A previously well boy of 34 weeks' gestation presented aged 8 days with lethargy, abdominal distension, and bile stained vomiting. An infection screen was performed. Abdominal radiographs showed gaseous distension but no specific features of necrotising enterocolitis.

Enteral feeding was stopped and intravenous antibiotics commenced with flucloxacillin, netilmicin, and metronidazole. The cerebrospinal fluid contained only 4 white cells $/ \mathrm{mm}^{3}$, but $E$ faecium was isolated on culture. Blood cultures were negative. Antibiotic treatment was changed to ampicillin and gentamicin as the organism demonstrated only moderate sensitivity to penicillin, and resistance to chloramphenicol. Treatment was continued for 14 days, with metronidazole for the initial seven days. Feeds were successfully reintroduced after a week and the baby subsequently made satisfactory progress, with discharge aged 4 weeks.

CASE 2

A boy was born at 33 weeks' gestation with an antenatally detected gastroschisis. After surgical repair on day 1 progress was satisfactory until day 16, when an infection screen was performed and enteral feeding stopped because of pyrexia and lethargy. Antibiotic treatment was commenced with flucloxacillin and netilmicin. $E$ faecium was grown from blood cultures, but the cerebrospinal fluid contained no leucocytes and was negative on culture. Over the next three days the infant continued to have temperature instability, and the antibiotics were changed to penicillin, netilmicin, and metronidazole because of abdominal distension and the presence of dilated bowel loops on an abdominal radiograph. Repeat blood cultures 48 hours later were negative, and the baby's clinical condition improved, but he remained intermittently feverish. A further examination of his cerebrospinal fluid on day 24 revealed 803 white cells $/ \mathrm{mm}^{3}$. Direct culture yielded no bacterial growth, but on enrichment $E$ faecium was isolated. Treatment with chloramphenicol was commenced as the organism demonstrated high level resistance to penicillin and gentamicin. Repeat cultures of the cerebrospinal fluid on day 28 were sterile, and chloramphenicol was continued for 14 days. The baby's condition improved steadily, though cerebral ultrasound revealed ventricular dilatation which subsequently required insertion of a ventriculoperitoneal shunt. At 9 months his development was assessed as being within normal limits.

Enterococci are part of the normal adult and neonatal gut flora, and are frequent isolates from clinical specimens. They are generally of low pathogenicity and infrequently cause infection. A 10 year study of neonatal enterococcal bacteraemia revealed only four patients with meningitis, three of whom had central venous catheters in situ. ${ }^{1}$ Many studies of enterococcal sepsis do not differentiate between Enterococcus spp. Most 
infections are due to $E$ faecalis, with an estimated $5-10 \%$ due to $E$ faecium, ${ }^{2}$ and there are few reports of neonatal infection due to $E$ faecium. ${ }^{3}$

Both our cases had gastrointestinal pathology, representing the likely source of infection, and neither had a central venous catheter. Standard antibiotic treatment on many neonatal units consists of penicillin/ flucloxacillin and an aminoglycoside. Both infants initially received penicillin or flucloxacillin with netilmicin and metronidazole, which is not effective treatment for $E$ faecium infection. ${ }^{4}$

Enterococci should be considered as a possible cause of septicaemia and meningitis in newborn infants with gastrointestinal pathology. Isolates should be identified to species level and antibiotic sensitivities performed to detect resistance to standard treatment regimens.

\section{P BARKER P W BUSS N MARLOW Department of Neonatal Medicine, St Michael's Hospital, Southwell Street, Bristol BS2 8EG N M BROWN
M R MILLAR Department of Medical Microbiology, Bristol Royal Infirmary, Upper Maudlin Street,
Bristol BS2 $8 H W$}

1 Dobson SRM, Baker CJ. Enterococcal sepsis in neonates: features by age of onset and occurrence of focal infection. Pediatrics 1990; 85: 165-71.

2 Moellering RC. Emergence of enterococcus as a significant pathogen. Clin Infect Dis 1992; 14: significant

3 Coudron PE, Mayhall CG, Facklam RR, et al. Streptococcus faecium outbreak in a neonatal
intensive care unit. $\mathcal{f}$ Clin Microbiol 1984; 20: intensive

4 Moellering RC, Korzeniowski OM, Sande MA, Wennersten CB. Species-specific resistance to antimicrobial synergism in Streptococcus faecium and Streptococcus faecalis. $\mathcal{F}$ Infect Dis 1979; 140: 203-8.

\section{A variable dextrose delivery system for neonatal intensive care}

EDITOR,-Optimising glucose delivery and reducing fluctuations in blood glucose are important goals in the care of the sick newborn baby. Traditionally, neonates requiring intravenous treatment have received infusions of $10 \%$ dextrose. However, when water requirements are particularly high, as in the preterm baby with large insensible water losses or when water requirements are low, as in the baby in renal failure, the requirements for fluid volume and glucose delivery may clash, resulting in either hyperglycaemia or hypoglycaemia. In addition, both glucose tolerance and glucose requirements may show wide variation in the sick neonate, necessitating frequent changes in glucose concentrations. We describe a simple method allowing variable glucose delivery, independent of infusion volume, which also avoids the need to change solutions.

Two continuous infusions, containing 5\% and $50 \%$ dextrose respectively, are administered via a $\mathrm{Y}$ connector close to the baby. Mixing of the two concentrations in different proportions delivers a variable amount of glucose in a fixed volume or a fixed amount of glucose in a variable volume.

When $5 \%$ and $50 \%$ dextrose solutions are used the following equations describe the volume of each solution required per hour:

$$
\mathrm{X}=0 \cdot 11[(\mathrm{~A} \times \mathrm{B}) 1 \cdot 2-\mathrm{Z}]
$$

where $\mathrm{X}=$ rate of $50 \%$ dextrose infused in $\mathrm{ml} /$ hour, $\mathrm{Y}=$ rate of $5 \%$ dextrose infused in $\mathrm{ml} / \mathrm{hr}, \mathrm{Z}=$ total hourly infusion volume of $5 \%$ and $50 \%$ dextrose in $\mathrm{ml} /$ hour, $A=$ required dextrose infusion rate in $\mathrm{mg} / \mathrm{kg} / \mathrm{min}$, and $\mathbf{B}=$ patient's weight in $\mathrm{kg}$.

This formula is derived in the following way:

As $50 \%$ dextrose $=500 \mathrm{mg}$ dextrose per $\mathrm{ml}$ and $5 \%$ dextrose $=50 \mathrm{mg}$ dextrose per $\mathrm{ml}$

[1] $500 \mathrm{X}+50 \mathrm{Y}=$ total $\mathrm{mg}$ dextrose required per hou $=($ total $\mathrm{mg}$ dextrose required per minute) 60

[2] $\mathrm{X}+\mathrm{Y}=$ volume in $\mathrm{ml}$ of $50 \%$ and $5 \%$ dextrose $\begin{aligned} & \text { infused per hour } \\ = & Z\end{aligned}$

Equations [1] and [2] are simultaneous equations containing the same variables. Therefore dividing equation [1] by 50 gives:

[3] $10 \mathrm{X}+\mathrm{Y}=(\mathrm{A} \times \mathrm{B}) 1 \cdot 2$

and multiplying equation [2] by -1 gives:

[4] $-(\mathrm{X}+\mathrm{Y})=-\mathrm{Z}$

Adding equations [3] and [4] together gives:

[5] $9 \mathrm{X}=(\mathrm{A} \times \mathrm{B}) 1 \cdot 2-\mathrm{Z}$

therefore

[6] $\mathrm{X}=0 \cdot 11[(\mathrm{~A} \times \mathrm{B}) 1 \cdot 2-\mathrm{Z}]$

but

$\mathrm{Z} \quad \mathrm{X}+\mathrm{Y}$

and so

$\mathrm{Y}$

$=\mathrm{Z}-\mathrm{X}$

Therefore from a knowledge of desired glucose infusion rate in $\mathrm{mg} / \mathrm{kg} / \mathrm{min}$ (A), the baby's weight in $\mathrm{kg}(\mathrm{B})$ and the total hourly infusion volume in $\mathrm{ml} /$ hour $(\mathrm{Z})$, the respective infusion rates of $5 \%$ and $50 \%$ dextrose may be calculated, thus allowing independent alteration of glucose delivery and fluid volume. In our unit the calculations are run on a computer terminal situated in the intensive care area. The operator is required only to enter in the values for $\mathrm{A}, \mathrm{B}$, and $\mathrm{Z}$. The computer screen displays the calculated flow rates for the $5 \%$ and $50 \%$ dextrose lines and also informs the operator of the concentration of the mixed solution. Although we were initially concerned that high glucose concentrations might lead to an increase in the number of intravenous lines requiring replacement, we did not in fact see this. This may be due to a streaming effect of mixing glucose solutions close to the site of venous entry.

We use a $500 \mathrm{ml}$ bag of $5 \%$ dextrose running via a giving set and volumetric pump and $50 \%$ dextrose administered through a syringe pump. The system is easy to set up and after an initial introductory period was well understood by nursing and medical staff. Changes can be easily and quickly achieved simply by changing the rate of either or both infusions, without the need to change lines or solutions.

$$
\begin{array}{r}
\text { BUSHRA AL-RUBEYI } \\
\text { NEIL MURRAY } \\
\text { NEENA MODI } \\
\text { Department of Paediatrics and Neonatal Medicine, } \\
\text { Royal Postgraduate Medical School, } \\
\text { Hammersmith Hospital, } \\
\text { Du Cane Road, } \\
\text { London W12 ONN }
\end{array}
$$

\section{Method for securing umbilical lines}

EDITOR,-Several methods have been described for the adequate fixation of umbilical lines. ${ }^{1-5}$ All attempt to ensure that catheters are anchored securely once sited in the optimal position (above or below D12-L4). Accidental displacement of arterial lines must be avoided as this can lead to rapid blood loss.

Some techniques using adhesive tape applied directly to the infant's skin can cause problems. If vernix is present the tape may not stick. If the baby is very preterm removal of tape can lead to skin loss with subsequent risk of infection.

Some described methods are fiddly and time consuming especially in very tiny babies. Tying loops and purse strings can be difficult and gripping the catheter sufficiently to provide anchorage may lead to occlusion (particularly where fine lines are used).

We report a means of securing umbilical lines which avoids some of these pitfalls while remaining simple, rapid, and safe.

\section{Method (see figure)}

Step 1 - a 4/0 silk suture is looped through the skin of the cord close to the line to be secured. A knot is tied at the cord and the suture is cut leaving two threads about $5 \mathrm{~cm}$ in length parallel to the line.

Step $2-$ a $1 \mathrm{~cm} \times 1 \mathrm{~cm}$ length of adhesive tape is used to approximate the line and parallel threads.

Step 3 - the two ends of thread are folded down over the first piece of tape and a second similar piece of tape is used to fix the threads once more. Once the final position of the line is ascertained the tape and the line are pinched together to ensure security.

This method has proved useful for securing umbilical arterial and venous lines in particular but has also been used for the safe fixation of chest drains and dialysis catheters. It has the advantage of only requiring a single stitch, of avoiding tape adhered direct to the premature infant's skin, and of being easily adjusted should radiograph checks require this. The method has been widely used on this unit with babies of 23 weeks' gestation upwards and has proved consistently reliable.
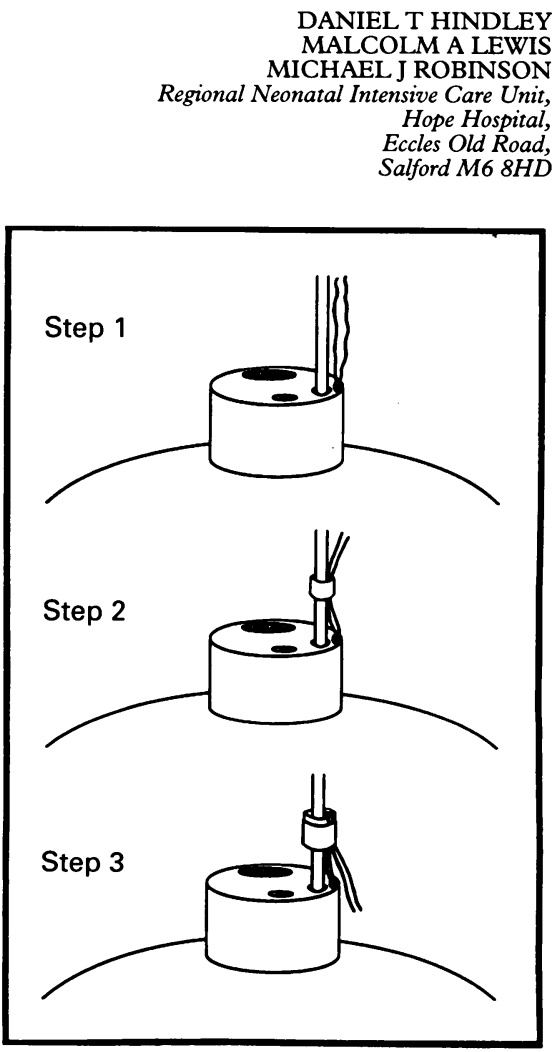